\title{
Escherichia coli Shiga-like Toxin Measurement
}

National Cancer Institute

\section{Source}

National Cancer Institute. Escherichia coli Shiga-like Toxin Measurement. NCI Thesaurus.

Code C154818.

The determination of the Escherichia coli shiga-like toxin present in a sample. 